\title{
IL-GLOBO (1.0) - development and verification of the moist convection module
}

\author{
Daniele Rossi, Alberto Maurizi, and Maurizio Fantini \\ Institute of Atmospheric Sciences and Climate, National Research Council, CNR-ISAC, Bologna, Italy \\ Correspondence to: Alberto Maurizi (a.maurizi@isac.cnr.it)
}

Received: 3 August 2015 - Published in Geosci. Model Dev. Discuss.: 28 September 2015

Revised: 16 February 2016 - Accepted: 19 February 2016 - Published: 26 February 2016

\begin{abstract}
The development and verification of the convective module of IL-GLOBO, a Lagrangian transport model coupled online with the Eulerian general circulation model GLOBO, is described. The online-coupling promotes the full consistency between the Eulerian and the Lagrangian components of the model. The Lagrangian convective scheme is based on the Kain-Fritsch convective parametrization used in GLOBO. A transition probability matrix is computed using the fluxes provided by the Eulerian KF parametrization. Then, the convective redistribution of Lagrangian particles is implemented via a Monte Carlo scheme. The formal derivation is described in details and, consistently with the Eulerian module, includes the environmental flux in the transition probability matrix to avoid splitting of the convection and subsidence processes. Consistency of the Lagrangian implementation with its Eulerian counterpart is verified by computing environment fluxes from the transition probability matrix and comparing them to those computed by the Eulerian module. Assessment of the impact of the module is made for different latitudinal belts, showing that the major impact is found in the Tropics, as expected. Concerning vertical distribution, the major impact is observed in the boundary layer at every latitude, while in the tropical area, the influence extends to very high levels.
\end{abstract}

\section{Introduction}

Long-range transport of atmospheric tracers plays an important role in several fields ranging from atmospheric composition and chemistry to climate change, with applications spanning from air pollution to natural or anthropogenic disaster management and assessment.
Lagrangian description is the natural framework for tracer dispersion modelling, and the use of Lagrangian particle dispersion models (LPDM) for both theory and application is very effective and widespread. In particular, Lagrangian models are used often to retrieve information about the sources contributing to the concentration at a specific location (known as "backtrajectories"). The consistent implementation of LPDM requires the careful consideration of all the processes involved in the atmospheric dispersion.

Depending on the geographical area and season, the redistribution of tracers released in the atmosphere can be largely affected by the vertical transport due to moist convection events. In particular, convection is very efficient in mixing the boundary layer with the free-troposphere air (Cotton et al., 1995) contributing to the long-range spread of local emissions.

Moist convection is widespread in the Earth's atmosphere where it displays a wide range of space- and timescales in response to the variability of environmental parameters, ranging from the sub-kilometre/tens-of-minutes of individual cumuli to hundred-of-kilometre/several days of mesoscale convective complexes (see e.g. Emanuel, 1994).

For all the scales smaller than or close to the grid size of the numerical models, explicit resolution is inadvisable, and numerical models resort to parametrization schemes.

A discussion of the theoretical issues and field of application of the different convective parametrizations is beyond the scope of this introduction - the interested reader is referred to Arakawa (2004) and references therein. In this work, a slightly modified Kain and Fritsch (1990, hereinafter $\mathrm{KF})$ scheme is adopted, which will be described briefly in Sect. 2. 
For the turbulent diffusion processes (that are predominant in the boundary layer), whose typical space- and timescales are small compared to the resolution of a general circulation model, a well-founded theoretical framework exists and allows for the formulation in terms of stochastic processes (Thomson, 1987). In contrast, mass-flux theories of moist convection do not provide sufficient details to implement stochastic models. Therefore, moist convection effects are simulated using particle redistribution mechanisms, which reproduces the expected mass fluxes obtained from an Eulerian convective scheme parametrization, usually via a Monte Carlo scheme (e.g. Forster et al., 2007).

While coupled chemistry and meteorology models (CCMMs) are now at the front edge of research in atmospheric composition studies (Baklanov et al., 2014), popular LPDMs are designed for offline usage and need to reconstruct necessary quantities that are not included in the normal output of meteorological models. In particular, offline recalculation of convective mass fluxes is needed, from quantities made available from the meteorological (Eulerian) model such as temperature and moisture, and is often performed with a mass-flux scheme different from the one that produces the meteorological output (see e.g. Forster et al., 2007), possibly leading to dynamical inconsistencies in the results.

To avoid this inconsistency, IL-GLOBO (Rossi and Maurizi, 2014) was designed as an online-coupled model that makes use of the full availability of Eulerian fields. In its first step of development, the vertical transport and dispersion of tracers were the result of the vertical advection and diffusion only. In the absence of a convective parametrization, explicit convection can occur, and thus some vertical transport of tracers were present in the previous version of the model. However, since the scales of convection are in the sub-kilometre range, any explicit representation of it at coarser resolution is bound to misrepresent most of those scales, and create updrafts that are incorrect in location and strength. Therefore the inclusion of a moist convection Lagrangian redistribution mechanism is essential to the completeness of the model. The IL-GLOBO moist convection module is developed consistently with the modified KF scheme adopted in GLOBO (Malguzzi et al., 2011) (see Sect. 2). With the online coupling this module benefits from the full availability of all meteorological variables at every time step.

In this paper the development of the online-coupled convective module of IL-GLOBO is presented and its features will be assessed through some application examples. In Sect. 2 the Eulerian convective parametrization is presented while the implementation of the Lagrangian scheme is described in Sect. 3 with emphasis on Eulerian consistency and providing full details of the constructive procedure. Verification of the scheme and some evaluation of the inclusion of convective effects in IL-GLOBO are presented in Sect. 4.

\section{The Kain-Fritsch scheme}

A convective parametrization that makes explicit use of the vertical fluxes of mass, the KF scheme, was adopted by the GLOBO developers, and in the present work, for its ready availability, ease of implementation and widespread use by the meteorological research community.

The original formulation, and its successive evolution, were presented in a series of papers (Fritsch and Chappel, 1980; Kain and Fritsch, 1990; Kain, 2004) to which the reader is referred for more details. Recent presentations of its performance in the simulation of meteorological events can be found e.g. in Liu and Wang (2011) and Bullock et al. (2015).

The scheme is based on a steady-state entrainingdetraining plume model and a closure based on release of convective available potential energy (CAPE). Three streams of mass are present: updraft and downdraft of the convecting ensemble, and a weak environmental flow (subsidence) that maintains the balance of mass at each model level.

The updraft is a detailed account of the thermodynamics of moist air and entrainment-detrainment of moisture and condensate at every level between cloud base and the cloud top. Briefly, mixtures of low-level air are tested for instability. Once a lifted condensation level (LCL) is identified, the parcel buoyancy at each upward level is computed, and an estimate of the kinetic energy gained by latent heat release obtained. The, as yet unspecified, upward mass flux is then fractionally increased/reduced by entrainment/detrainment of environmental air based on a buoyancy-sorting principle. The dilution of the originally unstable air with drier and cooler air from the environment reduces its buoyancy, up to an equilibrium level (LET) where the rising air has no more acceleration from thermodynamic processes. Upward of the LET, the rising air is decelerated until the remaining kinetic energy of the vertical motion is reduced to zero, which defines the top of the cloud.

Downdrafts are generated by re-evaporation of water condensate expelled by the rising motion. Environmental air is assumed to be entrained uniformly into the downdraft in a layer around cloud base, and detrained, again linearly in pressure, at lower levels. The empirical evidence for this structure is discussed at length in Kain (2004).

Only at this point are the dimensional mass fluxes determined by applying the closure assumption that requires at least $90 \%$ of the CAPE to be consumed by the ensemble of convective clouds. This finally determines the fraction of a grid box covered by the ensemble of clouds and the environmental subsidence needed to maintain the balance of mass at each level.

The tendencies of thermodynamic quantities to be returned to the model are spread over a "convective timescale" $\Delta T_{\mathrm{C}}$, ranging from half an hour to an hour, covered by several advective time steps $\Delta t$, so that each model time step only receives a fraction of the convective tendencies. 

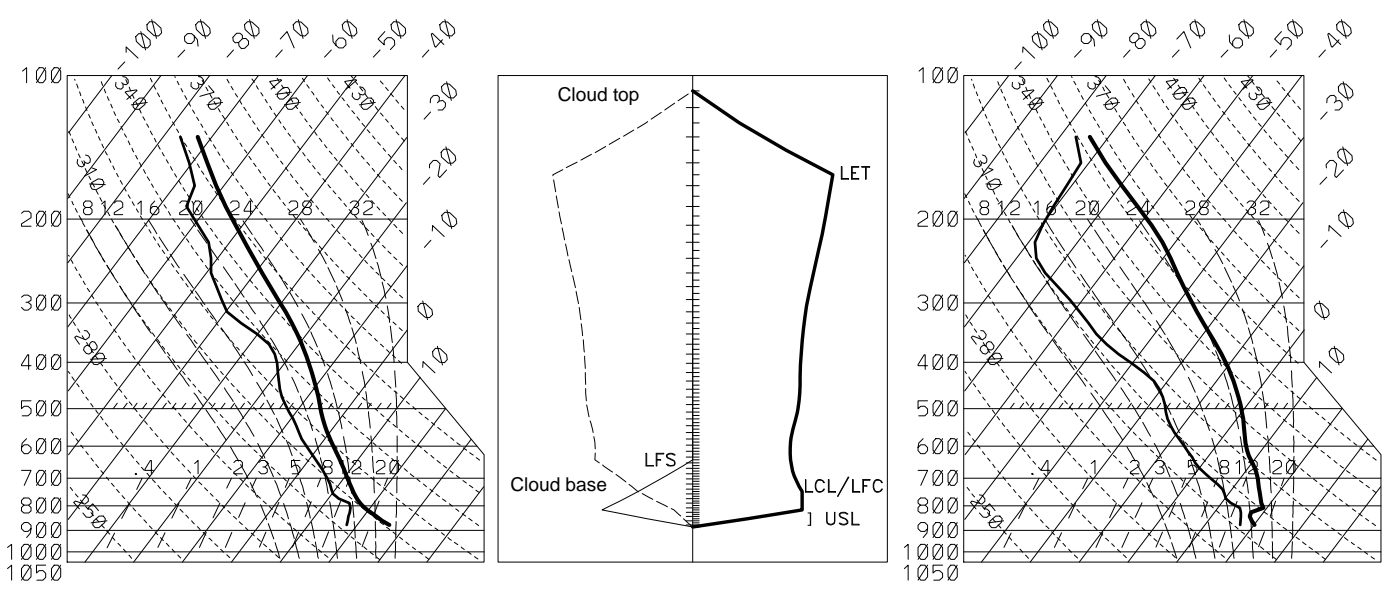

Figure 1. SkewT-logP thermodynamic diagrams for a deep tropical convective episode, before (left panel) and after (right panel) the action of convection as determined by the parametrization scheme: pressure on left axis in $\mathrm{hPa}$, temperature on right and top axes in ${ }^{\circ} \mathrm{C}$. Middle panel: profiles of vertical mass fluxes as computed by the convection scheme: updraft $\left(F^{\mathrm{u}}\right.$, thick solid line), downdraft $\left(F^{\mathrm{d}}\right.$, thin solid line), environmental subsidence ( $F^{\mathrm{e}}$, dashed) needed to maintain the balance of mass at each level (see Fig. 2 for definitions). Vertical coordinate for the middle panel is pressure, with the same scale indicated for left and right panels. Horizontal coordinate is $\mathrm{kg} \mathrm{s}^{-1} \mathrm{~m}^{-2}$ with arbitrary scale: the flux per unit area depends on the area attributed to the convective ensemble by the convection scheme. In this instance it is about $7 \%$ of the grid box. Also shown on the central axis are the locations of model levels. Significant levels for the convection computation are labelled on the graph. The air in the updraft source layer (USL) becomes saturated when raised to the lifted condensation level (LCL), and is unstable when further pushed to its level of free convection (LFC - at the same model level in this instance). Vertical acceleration of the rising air parcel ceases at the level of equilibrium temperature (LET) and vertical motion stops at cloud top. The convective downdraft begins at the level of free sink (LFS) and extends down to the ground.

An example of the effect of the scheme on an unstable atmospheric profile is shown in Fig. 1.

\section{Lagrangian implementation of the moist convection effects}

IL-GLOBO uses some of the quantities computed by the $\mathrm{KF}$ convective parametrization (see Sect. 2) to implement a Monte Carlo scheme (KF-MC) for the particle displacement, in a way similar to other LPDMs (Collins et al., 2002; Forster et al., 2007). All these schemes compute the displacement probability matrix (DPM) between levels making use of the entrainment and detrainment fluxes in updraft and downdraft. Additionally, in IL-GLOBO the environment effects (subsidence), that result from a mass balance, are directly included into the DPM, and therefore implemented using the MC scheme, without the need of a posteriori adjustment.

In the following, the same notation as in Fig. 1 of Rossi and Maurizi (2014) is used where NLEV $\sigma$-hybrid grid levels are indexed decreasing with height.

In the Eulerian model component, every $\Delta T_{\mathrm{C}}$ (or, in terms of time steps, every $n_{\mathrm{C}}$ advective time step $\Delta t$ ), the KF scheme checks for the conditions for the onset of convection and, if conditions are met, determines the evolution of the grid column for the whole $\Delta T_{\mathrm{C}}$. Entrainment and detrainment fluxes in both updraft $\left(f^{\mathrm{u} \varepsilon}, f^{\mathrm{u} \delta}\right)$ and downdraft $\left(f^{\mathrm{d} \varepsilon}\right.$, $f^{\mathrm{d} \delta}$ ) for each level (see Fig. 2), from the ground to the cloud

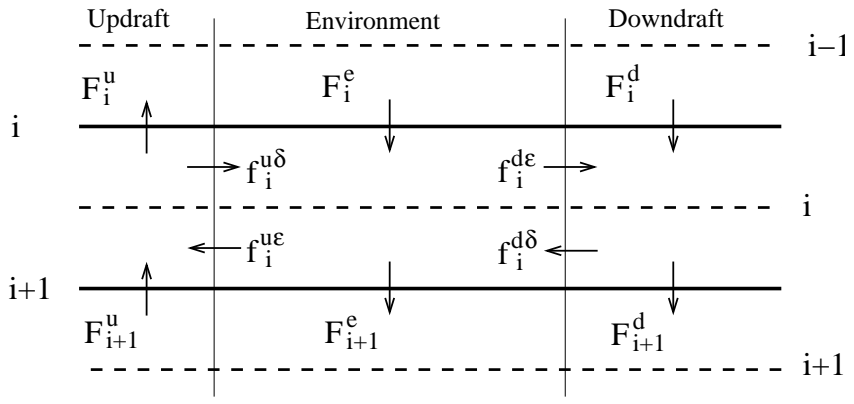

Figure 2. Schematic representation of fluxes involved in the KF scheme. Uppercase $F$ represent the fluxes between vertical levels (across level boundaries) while lowercase $f$ are the fluxes within a level that represent the exchange of mass between environment (e) and updraft (u) or downdraft $(\mathrm{d})$, respectively.

top, as computed by the KF scheme are made available to the Lagrangian model. With reference to Fig. 2, the following relationships hold for fluxes at $(f)$ and between $(F)$ levels:

$F_{i}^{\mathrm{u}}=F_{i+1}^{\mathrm{u}}+f_{i}^{\mathrm{u} \varepsilon}-f_{i}^{\mathrm{u} \delta}$

for the updraft (u) and

$F_{i+1}^{\mathrm{d}}=F_{i}^{\mathrm{d}}+f_{i}^{\mathrm{d} \varepsilon}-f_{i}^{\mathrm{d} \delta}$

for downdraft (d). The probability for a particle to be entrained in an updraft at level $i$ is expressed by the product of the probability to be entrained from the environment 
$\left(f_{i}^{\mathrm{u} \varepsilon} \Delta t / m_{i}^{\mathrm{e}}\right)$ and the probability that the particle resides in the environment $\left(m_{i}^{\mathrm{e}} / m_{i}\right)$, where $m_{i}^{\mathrm{e}}$ is the mass already present in the environment as opposed to the mass flowing through, in the convective ensemble, and $m_{i}$ is the total mass of the level $i$, giving

$p_{i}^{\mathrm{u} \varepsilon}=\frac{f_{i}^{\mathrm{u} \varepsilon} \Delta t}{m_{i}}$.

The probability that a particle captured by the updraft is detrained can be easily derived by rearranging Eq. (1) into

$\frac{F_{i}^{\mathrm{u}}}{F_{i+1}^{\mathrm{u}}+f_{i}^{\mathrm{u} \varepsilon}}+\frac{f_{i}^{\mathrm{u} \delta}}{F_{i+1}^{\mathrm{u}}+f_{i}^{\mathrm{u} \varepsilon}}=1$.

Noticing that the two terms are both positive by definition, the above relationship can be used to define the probability

$p_{i}^{\mathrm{u} \delta}=\frac{f_{i}^{\mathrm{u} \delta}}{F_{i+1}^{\mathrm{u}}+f_{i}^{\mathrm{u} \varepsilon}}$,

which is identically equal to 1 at the cloud top where $F_{i_{\text {top }}}^{\mathrm{u}}=$ 0 . The denominator $F_{i+1}^{\mathrm{u}}+f_{i}^{\mathrm{u} \varepsilon}$ of Eq. (4) is the flow entering the updraft volume (see Fig. 2) at level $i$ and that is available to detrainment process. The mass entering the level $i$ which is not detrained must flow to the upper level satisfying the continuity for the updraft (Eq. 1). In terms of probability, this is expressed by the complementary probability $\overline{p_{i}^{\mathrm{u} \delta}}=1-p_{i}^{\mathrm{u} \delta}$

$\overline{p_{i}^{\mathrm{u} \delta}}=\frac{F_{i}^{\mathrm{u}}}{F_{i+1}^{\mathrm{u}}+f_{i}^{\mathrm{u} \varepsilon}}$

which, combined with Eq. (5), gives back Eq. (1) confirming the consistency of the above definitions of the probability components.

Using the above definitions it is possible to build the full transition probability matrix for the updraft fraction. The probability that a particle moves due to the updraft motion from a level $i$ to a level $j<i$ is equal to the probability that the particle is entrained at level $i$ (Eq. 3) times the probability that it is detrained at level $j$ (Eq. 5) times the probability that it is not detrained between level $i$ and $j+1$ included. In formula:

$p^{\mathrm{u}}(j \mid i)=p_{i}^{\mathrm{u} \varepsilon \varepsilon} p_{j}^{\mathrm{u} \delta} \prod_{k=i}^{j+1}\left(1-p_{k}^{\mathrm{u} \delta}\right)$.

For the downdraft transition probability $p^{\mathrm{d}}$, a similar relationship holds. The probabilities $p^{\mathrm{u}}$ and $p^{\mathrm{d}}$ represent the upper and lower triangular components of the total convective transition probability matrix $\mathbf{p}^{\mathrm{c}}$ whose diagonal is defined by

$\mathbf{p}^{\mathrm{c}}(i \mid i)=1-p_{1}^{\mathrm{u} \varepsilon}\left(1-p_{i}^{\mathrm{u} \delta}\right)-p_{i}^{\mathrm{d} \varepsilon}\left(1-p_{i}^{\mathrm{d} \delta}\right)$.
The mixing produced by the convective motion (updraft and downdraft) needs to be balanced by the environment flux (subsidence) to conserve the mass. For the Eulerian part this is granted by the environmental flux computed in the KF scheme. In Lagrangian terms this is equivalent to maintaining a well-mixed state where the redistribution of mass is applied, and can be expressed in terms of DPM. This consistency is obtained by modifying the transition probability matrix $\mathbf{p}^{\mathfrak{c}}$ by imposing zero net flux at the interface between two model levels. At level $i$, the mass fluxes (assumed positive upward) across the two level interfaces $i$ (upper) and $i+1$ (lower) due to the sum of updraft and downdraft motion, are expressed in terms of probability as

$F_{i}^{\mathfrak{c}}=\sum_{k<i}\left[\mathbf{p}^{\mathrm{c}}(k \mid i) m_{i}-\mathbf{p}^{\mathrm{c}}(i \mid k) m_{k}\right]$

and

$F_{i+1}^{\mathrm{c}}=\sum_{k>i}\left[\mathbf{p}^{\mathrm{c}}(i \mid k) m_{k}-\mathbf{p}^{\mathrm{c}}(k \mid i) m_{i}\right]$

respectively. Thus, the mass conservation reads

$F_{i}^{\mathrm{c}}+F_{i}^{\mathrm{e}}=F_{i+1}^{\mathrm{c}}+F_{i+1}^{\mathrm{e}}$,

where $F^{\mathrm{e}}$ is the environment mass flux which is directed downward except in very peculiar cases (Kain et al., 2003) ${ }^{1}$. With the additional boundary condition

$F_{\mathrm{NLEV}+1}^{\mathrm{e}}=0$,

the environment flux can be computed iteratively through Eqs. (9)-(11). The effect of the environment flux at surface $i+1$ is to increase the transition probability from $i$ to $i+1$ while reducing the probability of the "null transition" (particle remains in the same model level). This results in the modification of the elements of the diagonal:

$p(i \mid i)=\mathbf{p}^{\mathrm{c}}(i \mid i)-\frac{F_{i+1}^{\mathrm{e}} \Delta t}{m_{i}}$

and sub-diagonal:

$p(i+1 \mid i)=\mathbf{p}^{\mathrm{c}}(i+1 \mid i)+\frac{F_{i+1}^{\mathrm{e}} \Delta t}{m_{i}}$.

The final DPM is then defined by

$p(j \mid i) \equiv \mathbf{p}^{\mathrm{c}}(j \mid i)$

for $j<i$ or $j>i+1$ and by Eqs. (13) and (14) for $j=i$ and $j=i+1$, respectively.

It is worth noting that $p$ is an Eulerian quantity that can be viewed as the linear operator acting on an initial concentration vector to give the concentration distribution after the

\footnotetext{
${ }^{1}$ The very unlike case of upward $F^{\mathrm{e}}$ is accounted for in the numerical code to avoid numerical inconsistencies.
} 
convection mixing. However, since $p$ is defined in terms of a finite time step $\Delta t$, it may become unstable (flux in one time step comparable to or larger than the mass of the level). In fact, $\mathrm{KF}$ use a reduced time step $\Delta t_{\mathrm{KF}}=\Delta T_{\mathrm{C}} / n_{\mathrm{KF}}$, with integer $n_{\mathrm{KF}}$, internally computed to maintain linear stability of the numerical scheme. Consistently, the same $\Delta t_{\mathrm{KF}}$ is used to compute the transition probability that will be iterated $n_{\mathrm{KF}}$ times using the MC scheme.

In order to implement the MC scheme, it is convenient to compute the cumulative transition probability matrix $\mathbf{P}$ as

$\mathbf{P}_{j, i}=\sum_{k=\mathrm{NLEV}}^{j} p(k \mid i)$.

The MC scheme is applied in grid columns affected by convection to the particles that are below the cloud top by extracting a random number $\chi$, uniformly distributed between 0 and 1 , and comparing its value to $\mathbf{P}_{j, i}, j=\mathrm{NLEV}, i_{\text {top }}$ until a $j_{f}$ is found so that $\chi<\mathbf{P}_{j_{f}, i}$. A position is then attributed to the particle within the arrival grid cell using the same $\chi$ number to interpolate linearly between the grid cell boundaries (Forster et al., 2007):

$\sigma_{p}=\sigma\left(j_{f}\right)+\left(\chi-\mathbf{P}_{j_{f}-1, i}\right) /\left(\mathbf{P}_{j_{f}, i}-\mathbf{P}_{j_{f}-1, i}\right) \Delta \sigma$.

The MC scheme is iterated $n_{\mathrm{KF}}$ times to obtain the final position after $\Delta T_{\mathrm{C}}$. Then, as for the tendencies of thermodynamic quantities in the Eulerian part, the total particle displacement is spread over the $n_{\mathrm{C}}$ advective time steps that cover the convective period.

\section{Model verification}

In order to identify the main features of the KF-MC scheme, to verify its implementation and to assess its impact on dispersion, some numerical experiments were performed.

A number of convective episodes were extracted from a model simulation performed using a horizontal regular grid of $1200 \times 832$ cells of $0.3^{\circ} \times 0.22^{\circ}$, that corresponds to a resolution of about $23 \mathrm{~km}$ at mid-latitudes in longitude, and a regular vertical grid of 50 points in the $\sigma$-hybrid coordinate. The advective time step used was $\Delta t=150 \mathrm{~s}$. The convective timescale is $T_{\mathrm{C}} \simeq 30 \mathrm{~min}$, i.e. the $\mathrm{KF}$ scheme is executed every $n_{\mathrm{C}}=11$ advective time steps.

\subsection{Displacement probability matrix}

An example selected in the tropical area around noon is shown in Fig. 3. The central part of the figure show the DPM for that specific event along with the vertical profiles of entrainment (bottom) and detrainment (left) fluxes in both updraft and downdraft. In this example the significant levels as defined in Fig. 1 are $\sigma_{\text {cloud top }}=0.41, \sigma_{\text {LET }}=0.47$, $\sigma_{\mathrm{LFS}}=0.79, \sigma_{\mathrm{LCL}}=0.93$ and the USL is a mixture $60 \mathrm{hPa}$ thick based on the ground. The two most likely transitions are:
1. the particle stays within the starting grid cell (highest values are found in the diagonal because most of the model grid cell is not influenced by convection);

2. the particle is transferred in the cell just below, due to the environment flux (high values in the matrix subdiagonal).

The other transitions are directly caused by convective motion and are consistently less likely, with probabilities in the range $10^{-2}-10^{-5}$. It is expected that finer model resolution would increase the ratio between the volume involved in convection to the total volume of some model columns. Updrafts generate transitions with highest likelihood for displacements from levels next to the ground to levels just below the cloud top, while the downdraft transitions are permitted only from levels between 0.8 and $0.9 \sigma$ to levels between 0.9 and $1.0 \sigma$. This reflects the hypotheses underlying the formulation of the KF parametrization.

\subsection{Algorithm verification}

In order to verify the consistency of the implementation, the distribution of initially well mixed particles were verified after convection to be still well mixed. This is performed in 1D-like configuration by selecting 12 convectively active grid columns, releasing $4 \times 10^{4}$ well-mixed particles in each and integrating the model for a full $\Delta T_{\mathrm{C}}$. It is found that the distribution after such integration remains well mixed within the same error interval used in Rossi and Maurizi (2014). However, this only provides a test of the numerical implementation and not of the theoretical formulation and correct calculation of $\mathbf{p}^{\mathrm{c}}$. In fact, in contrast to the formulation of a Lagrangian turbulent diffusion model for which well mixing provides a necessary and sufficient condition (Thomson, 1987), the well-mixed state in the present scheme is maintained by construction of the environmental flux, whether $\mathbf{p}^{\mathrm{c}}$ is correct or not. Therefore an independent verification is necessary. This is done by comparing the environment fluxes computed using the DPM from Eqs. (9), (10) and (11) to those provided by the KF scheme. Such a verification, performed for a number of convective episodes, confirms that within the roundoff error $\left(10^{-7}\right)$ Eulerian and Lagrangian KF fluxes are the same.

\subsection{Impact of KF-MC on dispersion}

The impact of convection on the particle dispersion in a fully 3-D experiment is considered. The aim is to assess the importance of the moist convection mechanism with respect to diffusion and advection. Simulations start on 11 March 2011. Particles are released and then dispersed for 6 days, and their position is sampled every hour. The source consists of $N_{p} \simeq 7.4 \times 10^{5}$ pairs of particles, each pair sharing the same initial position. Particles are released between $\sigma=1.0$ and $\sigma=0.9$ proportionally to the average vertical 


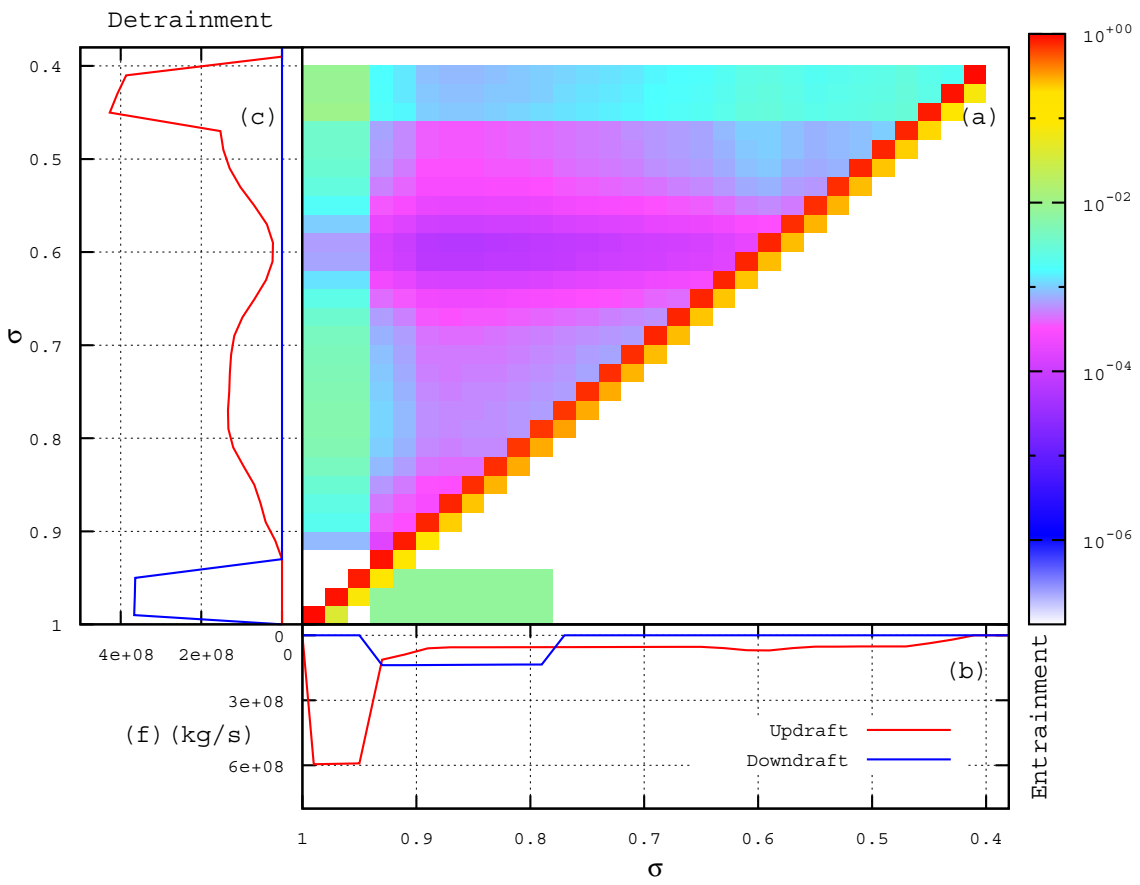

Figure 3. Example of Displacement Probability Matrix (DPM) and the fluxes generated by the convection mechanism, as function of the vertical $\sigma$-hybrid coordinate. Panel (a) displays the DPM with origins of displacement in the abscissa and destination in the ordinate. Bottom (b) and left (c) panels display entrainment and detrainment fluxes, respectively, for both updraft (red) and downdraft (blue).

density profile, and homogeneously distributed in the horizontal within three zonal areas: around the Equator, within the tropical area $\left(-15^{\circ},+15^{\circ}\right)$, and at mid-latitudes in the Northern Hemisphere and $\left(+30^{\circ},+60^{\circ}\right)$ and Southern Hemisphere $\left(-30^{\circ},-60^{\circ}\right)$. For each emission area, two different simulations were performed with the KF-MC switched on and off. Values of relative and absolute dispersion are shown in Fig. 4. Absolute dispersion is computed as

$\Delta_{a}^{2}=\frac{1}{2 N_{p}} \sum_{p=1}^{2 N_{p}}\left(x_{\mathrm{p}}-x_{\mathrm{p} 0}\right)^{2}$,

where $x_{\mathrm{p}}$ is a generic particle coordinate that can indicate both the particle vertical position (in Fig. 4 represented as the height above the model surface) and the horizontal distance along the Earth surface, and $x_{\mathrm{p} 0}$ is the starting position of the same particle. Relative dispersion is computed considering the ensemble of pair of particles sharing the same initial position and is defined as

$\Sigma_{r}^{2}=\frac{1}{N_{p}} \sum_{p=1}^{N_{p}}\left\langle\left(x_{\mathrm{p} 1}-x_{\mathrm{p} 2}\right)^{2}\right\rangle$,

where $x_{\mathrm{p} 1}$ and $x_{\mathrm{p} 2}$ are the position of each particle of the pair.

Results of the experiments, reported in Fig. 4, show that absolute dispersion is influenced by convection mainly at the Tropics, where the convective activity is more intense and the tropopause higher. Moreover, the effect is far more relevant on the vertical which is the direction directly influenced by the scheme. Concerning the relative dispersion, the moist convection scheme has a relevant impact on both the vertical and horizontal directions. The effect is important in all of the zonal areas but is still more pronounced at the Tropics. The larger impact on horizontal relative dispersion compared to the absolute dispersion can be explained by considering that as particles separate due to convection, they are captured by different horizontal structures that, in turn, rapidly decorrelate the motion of the two particles of the pair.

The effect on concentration is shown in Fig. 5, where the final concentration is displayed for vertical (Fig. 5, left panel) with and without moist convection scheme. For the horizontal Fig. 5, right panel, a difference map is shown. Particles were counted for intervals of equal size and normalized so that the starting concentration between 0.9 and $1.0 \sigma$ is around 1 . Figure 5 , left panel shows that moist convection has an important effect close to the surface in all the areas, with an enhanced effect at the Tropics. In the free troposphere, the effect is almost negligible except for the tropical area above $\sigma=0.4$ where the largest effect is observed. It is worth noting that, in the tropical area, particles reach high levels even in the simulation without convection although with a concentration smaller by a factor of 2 . Since the diffusivity only acts between 0.8 and $1 \sigma$ in the vast majority of cases, the vertical transport of particles producing the high concentration above $\sigma=0.4$ can be attributed mainly to vertical advection that 


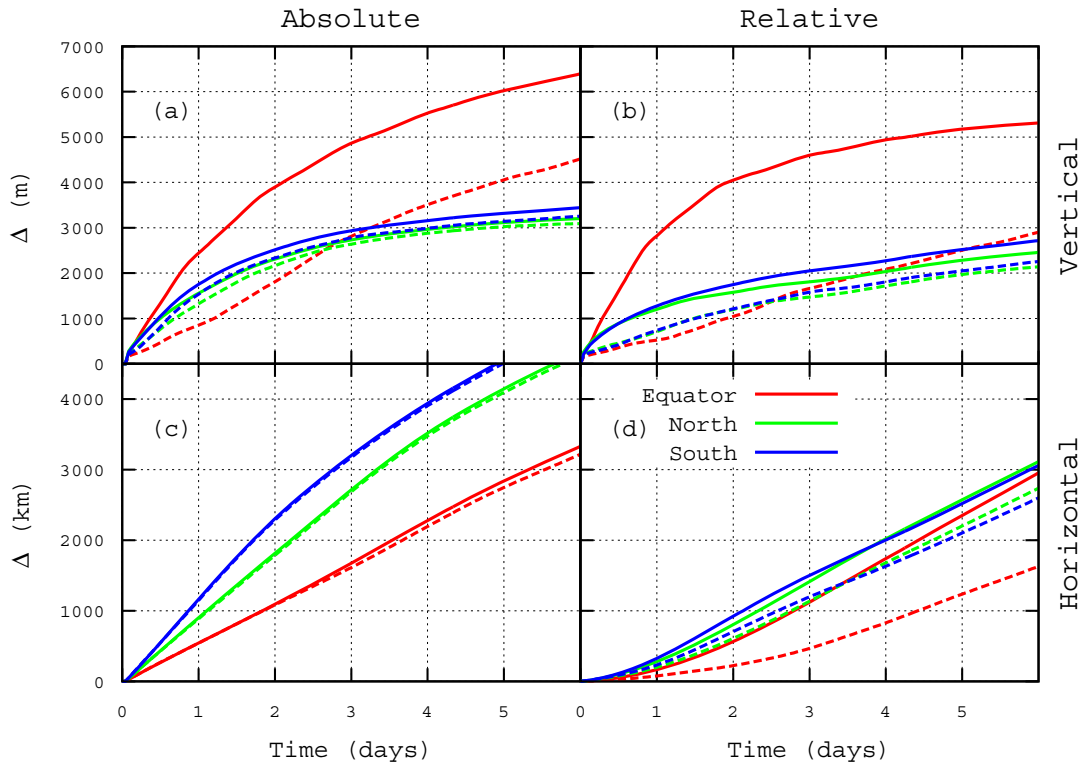

Figure 4. Vertical absolute dispersion (a), vertical relative dispersion (b), horizontal absolute dispersion (c), horizontal relative dispersion (d). Notice that panels (b) and (d) share the same $y$ axis with panels (a) and (c), respectively. Continuous lines refer to experiments with the MC convection scheme active, while the dashed lines mark experiments made without it. Line colours indicate the tropical distribution (red), northern middle-latitude distribution (green), southern middle-latitude (blue), respectively. Absolute and relative dispersion are defined by Eqs. (18) and (19).

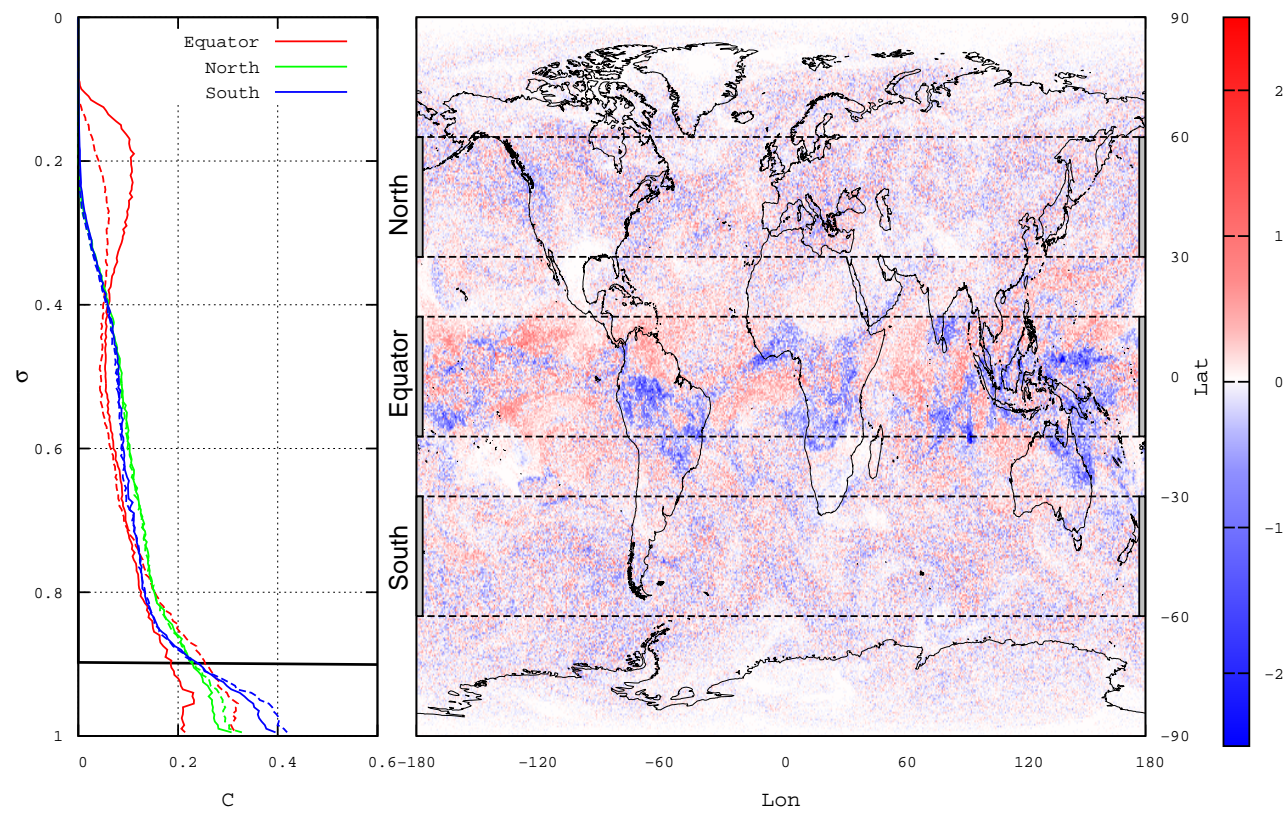

Figure 5. Left: vertical concentration profiles (in arbitrary units), as function of $\sigma$. Colours and line-types have the same meaning as in Fig. 4 . In addition, the top of the release region $(\sigma=0.9)$, is displayed as a bold solid line. Right: difference map (with convection minus without convection) of vertically integrated particle number at the end of the simulation for all the three emission subdomains (initial emission area as labelled in the figure: north, Equator, south). Details on the concentration normalizations for both panels can be found in Sect. 4.3. 
results from large-scale convergence with minor contribution from horizontal advection and orographic effects.

Figure 5, right panel, displays the map of differences of the vertically integrated number of particles between simulations with and without Lagrangian convection scheme. Particles are sampled for each $0.6^{\circ} \times 0.43^{\circ}$ column and the difference is normalized with respect to the initial number of particles per bin. For the case of release in the tropical area, it can be noted that areas of strong depletion are surrounded by relatively larger areas where the difference is weakly positive. The structure of convective updrafts (see e.g. Fig. 1) is such that most of the upward-moving mass comes from the lowest levels of the atmosphere (below cloud base) and is returned to the environment in the upper troposphere, in the strong outflow at the top of the cloud, while areas of weak subsidence surround the updrafts. Particles released in the extratropical regions (north and south) display different qualitative behaviour showing smaller-scale features with respect to those released at the Tropics, in agreement with the expected horizontal scales of convective cells.

\section{Conclusions}

A Lagrangian transport scheme for moist convection is implemented online in IL-GLOBO in parallel with the integration of the Eulerian model. This gives the Lagrangian scheme direct access to all the prognostic variables without any need for additional diagnostics and ensures full consistency of the DPM with the parametrization scheme. As a consequence, the Lagrangian and Eulerian descriptions of tracer dispersion in the coupled model are equivalent, as is expected on theoretical grounds.

This aspect differs from the approach of other models found in the literature. The quantities used in those cases to advect and diffuse Lagrangian particles are diagnosed from the meteorological thermodynamics profiles with parametrizations that may differ from that of the meteorological model, making the Eulerian-Lagrangian consistency hard to realize.

The consistency of the present scheme with the Eulerian quantities has been verified in a number of offline 1-D tests, where the model is shown to conserve the mass and reproduce the expected fluxes.

Global experiments with tracers released close to the surface at different latitudes show that the effects of the MC$\mathrm{KM}$ is strong and gives rise to large departures from the nonconvective version even at mid-latitudes. Vertical distribution displays again a larger difference at the Tropics. However, even with the KF-MC deactivated (but still activated in the Eulerian part, so generating the "correct" dynamics), at the Tropics some tracer is observed to reach very high altitudes. This is found to be a combined effect of advection, both vertical and horizontal.
The next step of the IL-GLOBO development will be the validation of the models against available data, for which appropriate data sets are scarce, as noted by e.g. Forster et al. (2007).

\section{Code availability}

The numerical code of the IL-GLOBO vertical moist convection module (Fortran 90) is released under the GPL and is available at the BOLCHEM website (http://bolchem.isac. cnr.it/source_code.do).

The software is packed as a library using autoconf, automake and libtool which allows for configuration and installation in a variety of systems. The code is developed in a modular way, permitting the easy improvement of physical and numerical schemes.

The GLOBO model is available upon the signature of an agreement with the CNR-ISAC Dynamic Meteorology Group (contact: p.malguzzi@ isac.cnr.it).

Acknowledgements. The authors would like to thank Piero Malguzzi for making the GLOBO model available and for making himself available for the explanation of the finer points of the numerics. The software used for the production of this paper (model development, model run, data analysis, graphics, typesetting) is free software. The authors would like to thank the whole free software community and, in particular, the Free Software Foundation (http://www.fsf.org) and the Debian Project (http://www.debian.org).

Edited by: O. Boucher

\section{References}

Arakawa, A.: The cumulus parameterization problem: Past, present and future, J. Climate, 17, 2493-2525, 2004.

Baklanov, A., Schlünzen, K., Suppan, P., Baldasano, J., Brunner, D., Aksoyoglu, S., Carmichael, G., Douros, J., Flemming, J., Forkel, R., Galmarini, S., Gauss, M., Grell, G., Hirtl, M., Joffre, S., Jorba, O., Kaas, E., Kaasik, M., Kallos, G., Kong, X., Korsholm, U., Kurganskiy, A., Kushta, J., Lohmann, U., Mahura, A., Manders-Groot, A., Maurizi, A., Moussiopoulos, N., Rao, S. T., Savage, N., Seigneur, C., Sokhi, R. S., Solazzo, E., Solomos, S., Sørensen, B., Tsegas, G., Vignati, E., Vogel, B., and Zhang, Y.: Online coupled regional meteorology chemistry models in Europe: current status and prospects, Atmos. Chem. Phys., 14, 317-398, doi:10.5194/acp-14-317-2014, 2014.

Bullock, O. R. j., Alapaty, K., and Herwehe, J. A.: A dynamically computed convective time scale for the Kain-Fritsch convective parameterization scheme, Mon. Weather Rev., 143, 2105-2120, doi:10.1175/MWR-D-14-00251.1, 2015.

Collins, W. J., Derwent, R. G., Johnson, C. E., and Stevenson, D. S.: A comparison of two schemes for the convective transport of chemical species in a Lagrangian global chemistry model, Q. J. Roy. Meteor. Soc., 128, 991-1009, 2002. 
Cotton, W. R., Alexander, G. D., Hertenstein, R., Walko, R. L., McAnelly, R. L., and Nicholls, M.: Cloud venting - A review and some new global annual estimates, Earth Science Review, 39, 169-206, 1995.

Emanuel, K. A.: Atmospheric Convection, Oxford Univ. Press, New York Oxford, USA, 1994.

Forster, C., Stohl, A., and Seibert, P.: Parameterization of Convective Transport in a Lagrangian Particle Dispersion Model and Its Evaluation, J. Appl. Meteorol. Clim., 46, 403-422, 2007.

Fritsch, J. M. and Chappel, C. F.: Numerical prediction of convectively driven mesoscale pressure systems. Part I: convective parameterization., J. Atmos. Sci., 37, 1722-1733, 1980.

Kain, J. S.: The Kain-Fritsch Convective Parameterization: An Update, J. Appl. Meteorol., 43, 170-181, 2004.

Kain, J. S. and Fritsch, J. M.: A one-dimensional entrainingdetraining plume model and its application in convective parameterization, J. Atmos. Sci., 47, 2784-2802, 1990.

Kain, J. S., Baldwin, M. E., and Weiss, S. J.: Parameterized Updraft Mass Flux as a Predictor of Convective Intensity, Weather Forecast., 18, 106-116, 2003.
Liu, H. and Wang, B.: Sensitivity of regional climate simulations of the summer 1998 extreme rainfall to convective parameterization schemes, Meteorol. Atmos. Phys., 114, 1-15, doi:10.1007/s00703-011-0143-y, 2011.

Malguzzi, P., Buzzi, A., and Drofa, O.: The Meteorological Global Model GLOBO at the ISAC-CNR of Italy Assessment of $1.5 \mathrm{Yr}$ of Experimental Use for Medium-Range Weather Forecasts, Weather Forecast., 26, 1045-1055, doi:10.1175/WAF-D11-00027.1, 2011.

Rossi, D. and Maurizi, A.: IL-GLOBO (1.0) - integrated Lagrangian particle model and Eulerian general circulation model GLOBO: development of the vertical diffusion module, Geosci. Model Dev., 7, 2181-2191, doi:10.5194/gmd-7-2181-2014, 2014.

Thomson, D. J.: Criteria for the selection of stochastic models of particle trajectories in turbulent flows, J. Fluid Mech., 180, 529$556,1987$. 